\title{
Endobronchial brachytherapy in the treatment of malignant lung tumours
}

\author{
J.A. Escobar-Sacristán*, J.I. Granda-Orive*, T. Gutiérrez Jiménez*, J.M. Delgado*, A. Rodero Baños*, \\ R. Saez Valls*
}

Endobronchial brachytherapy in the treatment of malignant lung tumours. J.A. Escobar-Sacristán, J.I. Granda-Orive, T. Gutiérrez Jiménez, J.M. Delgado, A. Rodero Baños, R. Saez Valls. C) ERS Journals Ltd 2004.

ABSTRACT: A prospective study was made to assess the short-term clinical and endoscopic response to high-dose-rate endobronchial brachytherapy (HDREB) in patients with malignant endobronchial tumours.

From July 1995 to May 2000, 288 HDREB sessions were carried out on 81 patients. The mean patient age was 61.57 yrs (range 34-82); males were predominant $(87.65 \%)$. Tumours were primary in 76 patients $(93.82 \%)$ and metastatic in five patients $(6.18 \%)$. The inclusion criteria were malignant endobronchial tumour and either palliative treatment for incurable disease or intent-to-cure treatment for residual malignancy on the bronchial resection surface after surgery or an inoperable tumour. The exclusion criteria were as follows: impediments to catheter placement, expected survival $<2$ months, Karnofsky index $<\mathbf{6 0}$, or absence of informed consent. The clinical response of a symptom was categorised as complete (disappearance of the symptom), partial (less than complete) or absent. The endoscopic response was considered to be complete if lesions disappeared and biopsy findings remained negative 1 month after the last radiation session; partial if lesions improved to some extent, but the biopsy findings were positive; and absent if there was no change in relation to baseline. The technique consisted of delivering high-dose irradiation from an $\mathrm{Ir}^{192}$ source to a target volume using one or two endobronchial catheters inserted under optical or video bronchoscopic guidance. Four sessions were scheduled at weekly intervals and $500 \mathrm{cGy}$ was applied per session over a length of 1-9 cm, measured $0.5-1 \mathrm{~cm}$ from the centre of the source.

In total, $85 \%$ of the symptoms analysed (haemoptysis, cough, dyspnoea, expectoration, and stridor) disappeared with HDREB, which was categorised as a complete response. The endoscopic response was complete in $56.79 \%$ of patients, partial or less than complete in $40.74 \%$ and absent in $2.46 \%$. One major complication occurred (bronchial fistula $1.2 \%$ ), but no lethal haemoptysis. Minor complications (pneumonitis, bronchospasm and bronchial stenosis) each occurred in one patient $(1.2 \%)$.

High-dose-rate endobronchial brachytherapy is a good palliative treatment for endoluminal lung neoplasms, effectively alleviating symptoms and endoscopic evidence in many cases with an acceptable rate of complications. High-dose-rate endobronchial brachytherapy can be carried out as an intent-to-cure procedure in highly selected cases. Eur Respir J 2004; 24: 348-352.
*Pulmonology Service and ${ }^{\#}$ Oncological Radiotherapy Service, Defence Military Hospital, Madrid, Spain.

Correspondence: J.I. de Granda-Orive

Pulmonology Service

Defence Military Service

C/ Glorieta del Ejercito s/n

28025 Madrid

Spain

Fax: 34914228201

E-mail: igo01m@saludalia.com

Keywords: Brachytherapy endobronchial high-dose rate lung cancer

Received: December 112002

Accepted after revision: April 292004
The term brachytherapy, from the Greek word brachy meaning "short", is applied to an irradiation technique designed to minimise the distance between the radioactive source and the target tumour volume. High-dose-rate (HDR) brachytherapy is a proven palliative therapeutic option. In highly selected cases, it can fully alleviate airway obstruction by malignant primary or metastatic tumours [1-3]. Highdose-rate endobronchial brachytherapy (HDREB) relieves the symptoms of endobronchial tumour, including spirometric indices and exercise tolerance, increasing the ventilation/perfusion ratio and reducing airway obstruction [4]. A clear regression in endobronchial lesions is observed after treatment.

Only $25 \%$ of malignant lung tumours can be removed surgically [1]. The remainder must be managed with other treatment modalities, which may also be used as adjuvants to surgery.
External radiotherapy is often used, but tumour recurrence is common and re-treatment is difficult because healthy tissue shows little tolerance for high doses of radiation. In light of these limitations of external irradiation, endobronchial radiotherapy, also known as internal irradiation, is advantageous because it concentrates an effective dose in the area of the bronchial lesion, thus curtailing complications as a result of irradiation of healthy tissue $[1-3,5-10]$.

Another advantage of HDREB is that it reduces treatment time. The procedure can be carried out with a minimal hospital stay, thus reducing hospitalisation costs together with the risk of catheter displacement and radiation exposure $[1,3]$.

The International Commission of Radiation Units defines HDR as the application of more than $20 \mathrm{cGy} \cdot \mathrm{min}^{-1}$ (1 $\mathrm{rad}=1 \mathrm{cGy})$ [11].

The current authors report the short-term results of a 
prospective study of HDREB in relieving the symptoms of malignant endobronchial tumours, using a protocol proposed by the Pulmonary Service, Defence Military Hospital, Madrid, Spain [12]. Clinical response was evaluated in terms of the symptoms of obstruction and regression of endobronchial lesions.

\section{Population and methods}

\section{Study population}

A prospective study was made to evaluate the short-term effectiveness of HDREB in the treatment of symptoms or residual tumour in primary and metastatic lung tumours.

From July 1995 to May 2000, 81 patients with malignant primary or metastatic endobronchial processes were treated. A total of 288 HDREB sessions were carried out. The mean patient age was 61.6 yrs (range 34-82) and males were clearly predominant (71 of 81 patients, $87.7 \%$ ). In the class of primary tumours, epidermoid carcinoma was by far the most frequent type with 48 tumours, followed by 11 adenocarcinomas, seven large-cell tumours, seven small-cell tumours, two nonsmall-cell carcinomas and one cylindroma. The metastatic lesions corresponded to three adenocarcinomas of the colon, one infiltrating ductal carcinoma of the breast and one endometrial carcinoma.

A total of 127 sites in 81 patients were treated. Two catheters were used in 17 patients.

Tumour sites were located mainly in the left main stem bronchus $(19.7 \%)$, right main stem bronchus $(17.32 \%)$ and right upper lobe bronchus $(17.32 \%)$. Other sites in the bronchial tree ranged in frequency from $4.72-11.02 \%$.

Inclusion criteria were as follows: 1) palliative treatment for patients with evidence of a malignant endobronchial tumour not susceptible to intent-to-cure treatment; and 2) intent-tocure treatment for patients with a residual malignant lesion on the bronchial resection surface after surgical tumour excision and inoperable malignant endobronchial tumours.

Exclusion criteria were as follows: 1) impediments to correct bronchoscopic catheter insertion; 2) expected patient survival $<2$ months; 3) Karnofsky index $<60$; and 4) informed consent not granted.

Endobronchial tumour change included ulcerating, fungating, fleshy, necrotic or polypoid types. The endobronchial lesion most frequently treated was submucosal infiltration, followed by mass-forming and papillary tumours. "No lesion" was referred to when there were malignant cells, but no evidence of tumour visual recognition of mucosal abnormalities in the tracheobronchial tree; in these cases, the cutting-bag area was irradiated.

\section{Methods}

Each patient was prepared for the procedure with oral codeine phosphate given $1 \mathrm{~h}$ before the procedure, followed by $5 \mathrm{mg}$ diazepam and $0.5 \mathrm{mg}$ atropine given $30 \mathrm{mins}$ before the procedure. An Olympus fibro- or videobronchoscope (fibrobronchoscope 1T 20 and 1 TR 20 and videobronchoscope T 200, 1 T 200 and T 240; Olympus, Hamburg, Germany) was introduced through the nostril and used to locate the tumour site. A 995-mm long by 2-mm section catheter (6F 083026; Nucleotron, Veenendaal, The Netherlands), marked with radiopaque material to facilitate fluoroscopic guidance, was positioned under radiographic guidance and the bronchoscope was removed. Two catheters were inserted in 17 patients. The metal guide was removed and a simulation probe was introduced (a metal probe with centimetre markings). Posteroanterior and lateral radiographs were used to calculate the target area or treatment volume. After completing the simulation, the patient was introduced into a lead-sheathed chamber. The catheter positioned in the bronchial tree was used to deliver HDR radiation to the target area from an iridium ${ }^{192}$ source with Microselectron afterloading (Nucleotron).

No consensus has been reached regarding the total dose and dose fractionation in endobronchial brachytherapy. The therapeutic protocol of the current authors' centre consists of sessions carried out at 1-week intervals in which a total dose per session of $500 \mathrm{cGy}$ was applied over a distance of $1-9 \mathrm{~cm}$, measured $0.5-1 \mathrm{~cm}$ from the centre of the source.

As part of an integral approach to cancer treatment, HDREB was used in conjunction with other procedures, which are summarised in table 1. Treatment was individualised for each patient in relation to tumour stage.

In nine patients, endobronchial brachytherapy preceded other treatments that were given for recurrence. The 15 patients in whom brachytherapy was the initial treatment included: patients with tumour recurrence or persistence of tumoural cells on the bronchial resection margins; patients with poor respiratory function and bronchial obstruction; and patients with a short life expectancy and obstructive pneumonia.

The predominant clinical manifestations before treatment were as follows: cough $(n=34,41.97 \%)$; haemoptysis $(n=24$, $29.62 \%$ ); expectoration without haemoptysis $(\mathrm{n}=8,9.87 \%)$; dyspnoea $(n=24,29.62 \%)$; and stridor $(n=7,8.64 \%)$. The main objective of the study was to determine if HDREB relieved the symptoms of endobronchial tumour with an acceptable rate of complications. There was no intention to quantify the degree or duration of palliation. Endoscopic evidence of the regression of lesions is the best indicator of a positive response to treatment. Consequently, the criteria of therapeutic response were clinical improvement in symptoms, and bronchoscopic evidence of the reduction or disappearance of endobronchial histological lesions. The clinical response was categorised in terms of the remission of previous symptoms as complete, less than complete (partial) or absent. Bronchoscopy was performed 1 month after the last HDREB session. The response to HDREB was judged to be complete when there was no endoluminal pathology or signs of tracheobronchial wall infiltration, as confirmed by biopsy. The response was considered partial when the lesions and obstruction had decreased, but tumoural infiltration persisted. Nonresponse existed when the pathology remained unchanged after treatment.

Two catheters were used in 17 patients with multiple tumour sites in the bronchial tree. Each catheter was

Table 1. - Treatments administered to the studied patients in addition to high-dose-rate brachytherapy

\section{Treatments}

Chemotherapy+external irradiation

$19(23.45)$

Initial brachytherapy

$15(18.51)$

External irradiation

$13(16.04)$

Surgery+external radiation+chemotherapy

$10(12.34)$

Surgery

Surgery+external irradiation

Chemotherapy

External irradiation+prosthesis+laser

$8(9.87)$

7 (8.64)

$7(8.64)$

$1(1.23)$

External irradiation+laser

$1(1.23)$

Data are presented as $n(\%) . n=81$. 
introduced into a nostril, positioned distally and crossed to deliver the maximum dose to the disease sites.

Patients were scheduled for follow-up 1 month after the last HDREB session. Bronchoscopy, biopsy of the treatment zone and bronchoaspiration for cytological study were performed.

A descriptive statistical study was made of the sample. Proportions of repeated observations were compared with McNemar's symmetry test. A value of $\mathrm{p}<0.05$ was accepted as significant $(95 \%$ confidence interval).

\section{Results}

A complete clinical response was observed in $84.53 \%$ of the symptoms analysed (table 2). From 24 patients who had haemoptysis at baseline, only one patient had haemoptysis after HDREB and it was very scant. This was equivalent to $95.83 \%$ palliation $(\mathrm{McNemar}$ test $\mathrm{p}<0.0001)$. Of 34 patients with coughing at baseline, coughing disappeared or returned to the pre-tumoural situation in $30(88.23 \%$; McNemar test $\mathrm{p}<0.0001)$. From 24 patients with dyspnoea at baseline, 18 $(75 \%)$ experienced considerable improvement or their dyspnoea disappeared $(\mathrm{McNemar}$ test $\mathrm{p}<0.0001)$. Of eight patients who had increased expectoration at baseline, expectoration decreased or disappeared in four $(50 \%$; McNemar test $\mathrm{p}=0.125)$. Stridor disappeared in all seven patients who suffered from it before treatment $(100 \%$; McNemar test $\mathrm{p}<0.05$ ).

The endoscopic response was complete in 46 patients $(56.79 \%$; table 3$)$. The response was partial or less than complete in 33 patients $(40.74 \%)$ and there was no response in two $(2.46 \%)$.

Complications were infrequent and included a fistula from the medial wall of the right main bronchus to an area of lymph node enlargement in the subcarinal mediastinum. The fistula did not produce mediastinitis or death. Other complications that occurred were bronchospasm $(n=1)$, posttreatment bronchial stenosis $(n=1)$ and pneumonitis in the area proximal to the treated bronchus $(n=1)$.

The complications in the current study were fewer in number and less important than those reported in other studies.

\section{Discussion}

It was found that HDREB relieved the symptoms of endobronchial tumours with a low rate of complications. However, the procedure is still under development. Although its indications seem to be clear, the total dose and its fractionation remain to be determined [1-3, 5, 8, 13].

Low-dose-rate (LDR) brachytherapy has been used, but a catheter must be left in place for 1-3 days, with the attendant risk of displacement thus requiring hospitalisation of the patient during treatment and surveillance [1, 3]. For this reason, LDR brachytherapy has fallen into disuse. An intermediate-dose-rate has also been used, which requires
Table 3.-Endoscopic results for the studied patients

\begin{tabular}{lccc}
\hline & Complete & Partial & No response \\
\hline Endoscopic response & $46(56.79)$ & $33(40.74)$ & $2(2.46)$ \\
\hline
\end{tabular}

Data are presented as $\mathrm{n}(\%) . \mathrm{n}=81$.

keeping the catheter in place for hours, but does not require prolonged hospitalisation [3].

Patients with nonresectable primary bronchogenic carcinoma, recurrence or metastatic carcinoma of the airways are potential candidates for HDREB [1-3]. Some studies have reported cures in highly selected cases [1-3, 9, 12, 14-16].

The current authors treated 10 patients with bronchial amputation stumps and recurrence or persistence of tumour cells after surgery with intent-to-cure. HDREB was the first but not the only treatment used. The mean recurrence time in these patients was 8 months. It is believed that early HDREB should be indicated in tumours in which malignant cells are found on the resection margins after surgery, since the outcome depends on prompt treatment. Patient 11 achieved a local disease-free interval of 21 months with HDREB of the stump 1 month after surgery, reinforced by external radiotherapy. Some authors prefer to use the term "prolonged palliation" instead of "intent-to-cure". However, the results suggest that the possibility of a cure should not be overlooked.

It was found that symptoms of airway obstruction improved with HDREB in $84.53 \%$ of the studied patients. A clinical response was elicited in $95.83 \%$ of patients with haemoptysis, $88.23 \%$ of patients with cough and $50 \%$ of patients with expectoration. The two symptoms most directly related with obstruction, stridor and dyspnoea had a clinical response of $100 \%$ and $75 \%$, respectively. The current findings confirm that symptoms respond well to brachytherapy [14, 17], with haemoptysis being the symptom that most often disappears [14, 15, 17-20]. Dyspnoea improved in $75 \%$ of patients, as has also been reported in other studies $[14,15$, 17-19].

Response to HDREB was also evaluated by the endobronchial changes identified by bronchoscopy. In the patients treated with HDREB, all endobronchial changes disappeared in $46(56.79 \%)$, some endobronchial changes persisted in 33 $(40.74 \%)$ and no endobronchial changes were observed in two $(2.46 \%)$. In the follow-up biopsy of the target area, the endoscopic evidence was always better than that observed in the last brachytherapy session in all patients who responded. An overall response of $74-87 \%$ has been reported [14, 16, 19]. In the current study, a complete or partial response was observed in 79 patients $(97.53 \%)$.

The causes of variability in the endoscopic response are unclear, but a relationship with the extrabronchial size of the tumour has been postulated [19], suggesting that tumours $>5 \mathrm{~cm}$ in diameter have a less satisfactory response. It has been remarked that patients with extrinsic compression by an extrabronchial tumour are poor candidates for HDREB [13, 14]. Some physicians have omitted oat-cell tumours from their

Table 2. - Clinical results for the studied patients

\begin{tabular}{lccccc}
\hline & Haemoptysis & Cough & Dyspnoea & Expectoration & Stridor \\
\hline Clinical response & & & & & \\
Pre-treatment & 24 & 34 & 24 & 7 & 0 \\
Post-treatment & 1 & 4 & 6 & $<$ & 0.125 \\
p-value & $<0.0001$ & $<0.0001$ & $<0.0001$ & $<0.05$ \\
\hline
\end{tabular}

Data are presented as $n$. $n=81$. 
studies [1], but the current authors and others have not $[9,14$, $15,18,21]$. The current authors evaluated patients endoscopically with oat-cell tumour after chemotherapy and external radiotherapy. Residual endobronchial disease was treated with brachytherapy.

Few studies to date have investigated HDREB in combination with external radiotherapy or separately [18, 22, 23]. In the current authors' opinion, external radiotherapy and internal radiotherapy (brachytherapy) are complementary procedures. In a prospective randomised study of the effectiveness of endobronchial brachytherapy in enhancing the effect of external radiotherapy, it was concluded that, in patients with inoperable endobronchial tumour, HDREB increases tumour control when combined with external radiotherapy, but does not significantly prolong survival [24].

Of five metastatic tumours treated, a complete response was obtained in one patient with an endometrial metastasis and a partial response in three patients with metastases of adenocarcinoma of the colon and one with metastases of infiltrating ductal carcinoma of the breast.

A total of 11 patients underwent re-treatment. One of these patients had the only major complication: a fistula from the medial wall of the right main bronchus to a subcarinal tumour. Complications increased with the treatment of recurrences [1]. The current authors agree with COTTER et al. [25] that the response to treatment and complications depends on the dose delivered.

The procedure was well tolerated by all patients and each session required $24 \mathrm{~h}$ of hospitalisation. No catheter had to be removed as a result of intolerance in any patient and no treatment was discontinued for catheter-placement problems. These advantages confirm reports in the literature regarding shorter hospital stay, better tolerance and less catheter displacement [1, 3].

Although no consensus has been reached regarding optimal dose and fractionation, a protocol of four sessions scheduled at weekly intervals was used in the current study. A total dose of 2,000 cGy was given, except for a few cases in which larger doses were given in fewer sessions. With regards to the distance and depth of radiation, these were varied because it was thought that the complications' rate could be related to the thickness of the bronchi wall. This is the reason why the distance was varied and, with that schedule, low complication rates were achieved. This protocol reduced radiation toxicity and potentially severe complications, such as massive haemoptysis and fistulas, which are directly linked to the radiation dose to the bronchial wall $[1,5,18,25]$. The frequency of major complications (haemoptysis and fistulas) ranged from $0-42 \%$ in the earliest studies [1]. The risk depended on the endobronchial site of the tumour and its relationship with large vessels. The highest risk was associated with HDREB applied to the bronchus of the right upper lobe and left upper lobe, which can be affected by tumour infiltration during the natural progression of the neoplasm $[1,5,8,19]$, particularly in the case of recurrences [19]. Later studies concluded that the use of a less aggressive technique would reduce the frequency of major complications, some of which would be as a result of progression of the neoplasm [3, $14,17,18,21,26]$. Other complications include pneumothorax, bronchospasm, bronchial stenosis and postirradiation pneumonitis $[1,5,6,14,15,27]$. The complications that occurred in the current study included one major complication (bronchial fistula) and three minor complications (bronchospasm, bronchial stenosis and pneumonitis). Pneumonitis is an unusual complication considering the steep decay of the irradiation. It is thought that it could be because, in this case, two endobronchial catheters were used and these were inserted through different bronchi involving the tumour with healthy parenchyma. These complications are viewed as being clearly related with fractionation and the dose per session.

Few studies, other than that of TREDANIEL et al. [28], have demonstrated that HDREB increases survival in patients with malignant endobronchial processes. These authors claim that HDREB monotherapy, in strictly selected cases of small tumours limited to the bronchial lumen, can increase survival and response duration by producing a complete remission of the tumour; however, the authors remark that the same survival would be achieved with more conventional treatment. HUBER et al. [26] reported a nonsignificant increase in survival with HDREB and external radiotherapy and KELLY et al. [29] recently cited an improvement in symptoms in relation to endoscopic response and increased survival.

Although there has been little experience with HDREB after sensitisation to chemotherapeutic agents, some authors recommend this procedure [30]. The current authors have begun to treat patients with lung cancer using HDREB after sensitisation with Taxotere in an attempt to improve results.

\section{Conclusions}

HDREB is a good palliative treatment for endoluminal neoplasms of the lungs, either alone or in combination with other procedures. It can be carried out as an intent-to-cure procedure for highly selected tumours confined to the endobronchial surface, persistence of malignant cells in bronchial amputation stumps or small tumours in a bronchus accessible to bronchoscopy. It also has a good tolerance and low complication rate.

A high-dose-rate endobronchial brachytherapy protocol of four sessions spaced at 1-week intervals with a $500 \mathrm{cGy}$ dose per session seems to be a schedule that is effective and has low complications rates.

\section{References}

1. Villanueva AG, Lo TCM, Beamis JF. Endobronchial brachytherapy. Clin Chest Med 1995; 16: 445-454.

2. Nag S, Abitbol AA, Anderson LL, et al. Consensus guidelines for high dose rate brachytherapy in cervical, endometrial and endobronchial tumors. Int J Radiat Oncol Biol Phys 1993; 27: 1241-1244.

3. Hilaris BS, Porrazzo MS, Moorthy CR. Endobronchial radiation therapy. In: Wang KP, Metha AC, eds. Flexible bronchoscopy. Cambridge, Massachusetts Blackwell Science, 1995; pp. 275-287.

4. Goldman JM, Bulman AS, Rathemell AJ, Carey BM, Muers MF, Joslin CA. Physiological effect of endobronchial radiotherapy in patients with major airway occlusion by carcinoma. Thorax 1993; 48: 110-114.

5. Alberti WE. Endobronchial high dose rate brachytherapy. Int J Radiat Oncol Biol Phys 1993; 25: 753-755.

6. Hernández $\mathrm{P}$, Donath $\mathrm{D}$. High dose rate endobronchial brachytherapy in the treatment of bronchogenic carcinoma. Chest 1993; 104: 989-991.

7. Castella J, Puzo C, Mota S. Algunos avances en broncoscopia [Some advances in bronchoscopy]. In: Caminero Luna JA, Fernández Fau L, eds. Update SEPAR. Vol. 2. Barcelona, Prodisa, 1996; pp. 241-262.

8. Augun C, Blum JE. Treatment of unresectable lung cancer with brachytherapy. World J Surg 1995; 19: 823-827.

9. Tredaniel J, Hennequin C, Zalcman G, Walter S, Maylin CI, Hirsch A. Curitherapie endobronchique à haut débit of dose et sa place dans le désobstruction tumorale bronchique [High dose endobronchial curietherapy and its role in the relief of bronchial obstruction due to tumours]. Rev Mal Respir 1993; 10: 291-298. 
10. Taulelle M, De Faucal H, Benezet $\mathrm{O}$, et al. Traitement des cancer bronchiques obstructifs par curitherapie endobronchique à haut débit. Aspects techniques et resultats cliniques [Treatment of obstructive bronchial cancers with high dose, endobronchial curietherapy. Technical aspects and clinical results]. Rev Mal Respir 1993; 10: 347-351.

11. Speiser BL. Advantages of high dose rate remote afterloading systems: physics or biology. Int J Radiat Oncol Biol Phys 1991; 20: 1133-1135.

12. Escobar Sacristán JA, Granda Orive JI, Gutiérrez Jiménez T, Martínez Albiach JM, Fuentes Ramos M, Herrera de la Rosa A. Braquiterapia endobronquial con dosis elevada en tumores malignos del pulmón [High rate endobronchial brachytherapy in malignant lung tumours]. Arch Bronconeumol 1997; 33: 278-283.

13. Vergnon JM. Techniques interventionnelles en endoscopie bronchique [Interventional techniques in endobronchial endoscopy]. Encyclop Med Chir (Elsevier-Paris) Pneumologie, 6-000-M-10, 1997; pp. 11.

14. Speiser BL, Spratling L. Remote afterloading brachytherapy for the local control of endobronchial carcinoma. Int $J$ Radiat Oncol Biol Phys 1993; 25: 579-587.

15. Chang LF, Horvath J, Peyton W, Ling SS. High dose rate after loading intraluminal brachytherapy in malignant airway obstruction of lung cancer. Int $J$ Radiat Oncol Biol Phys 1994; 28: 589-596.

16. Zajac AJ, Kohn ML, Heiser D, Peters J. High dose rate intraluminal brachytherapy in the treatment of endobronchial malignacy. Radiology 1993; 187: 571-575.

17. Pish J, Villamena P, Harvey J, Rosemblatt E, Mishra S, Beattie E. High dose rate endobronchial irradiation in malignant airway obstruction. Chest 1993; 104: 721-725.

18. Gollins SW, Burt PA, Baber PV, Stout R. High dose rate intraluminal radiotherapy for carcinoma of the bronchus: outcome of treatment of 406 patients. Radiother Oncol 1994; 33: $31-40$

19. Bedwinek J, Petty A, Bruton C, Sofield J, Lee L. The use of high dose rate endobronchial brachytherapy to palliate symptomatic endobronchial recurrences of previously irradiated bronchogenic carcinoma. Int J Radiat Oncol Biol Phys 1991; 22: 23-30.

20. Nori D, Allison R, Kaplan B, Samala E, Osian A, Karbowitz $\mathrm{S}$. High dose rate intraluminal irradiation in bronchogenic carcinoma. Technique and results. Chest 1993; 104: 10061011.

21. Gauwitz M, Ellerbroek N, Komaki R, et al. High dose endobronchial irradiation in recurrent bronchogenic carcinoma. Int J Radiat Oncology Biol Phys 1992; 23: 397-400.

22. Furuta M, Tsukiyama I, Ohno T, et al. Radiation therapy for roentogenographically occult lung cancer by external beam irradiation and endobronchial high dose rate brachytherapy. Lung Cancer 1999; 25: 183-189.

23. Moghissi K, Bond MG, Sambrook RJ, Stephens RJ, Hopwood P, Girling DJ. Treatment of endotracheal or endobronchial by non-small cell lung cancer: lack of patients in an MRC randomized trial leaves key questions unanswered. Medical Research Council Lung Cancer Working Party. Clin Oncol ( $R$ Coll Radiol) 1999; 11: 179-183.

24. Huber RM, Fischer R, Hautmann H, Pöllinger B, Haeussinger N, Wendt $\mathrm{T}$. Does additional brachytherapy improve the effect of external irradiation? A prospective, randomized study in central lung tumors. Int $J$ Radiat Oncol Biol Phys 1997; 38: 533-540.

25. Cotter GW, Lariscy C, Ellingwood KE, Herbert D. Inoperable endobronchial obstructing lung cancer treated with combined endobronchial and external beam irradiation: a dosimetric analysis. Int J Radiat Oncol Biol Phys 1993; 27 531-535.

26. Huber RM, Fischer R, Hautmann $\mathrm{H}$, et al. Palliative endobronchial brachytherapy for central lung tumors. A prospective randomized comparison of two fractionation schedules. Chest 1995; 107: 463-470.

27. Speiser BL, Spratling L. Radiation bronchitis and stenosis secondary to high dose rate endobronchial irradiation. Int J Radiat Oncol Biol Phys 1993; 25: 589-597.

28. Tredaniel J, Hennequin C, Zalcman G, et al. Prolonged survival after high dose rate endobronchial radiation for malignant airway obstruction. Chest 1994; 105: 967-972.

29. Kelly JF, Delclos ME, Morice RC, Huaringa A, Allen PK, Komaki R. High-dose-rate endobronchial brachytherapy effectively palliates symptoms due to airway tumors: the 10years M.D. Anderson Cancer center experience. Int $J$ Radiat Oncol Biol Phys 2000; 48: 697-702.

30. Lee JS, Komaki R, Morice RC, et al. A pilot clinical laboratory trial of paclitaxel and endobronchial brachytherapy in patients with non-small cell lung cancer. Semin Radiat Oncol 1999; 9: Suppl. 1, 121-129. 